\title{
A Study on the Application of Internet of Things in Intelligent Security System
}

\author{
Ying Zhou, Pancheng Li \\ Jiangxi Police Institute, Nanchang City, Jiangxi Province, 330103, China
}

Keywords: Internet of things; Intelligent security system; Video surveillance; access control; Facial recognition

\begin{abstract}
The early Internet of things is a logistics network based on wireless sensor technology, but the connotation of the Internet of things has changed greatly with the continuous development of technology and application. The perfect combination of the Internet of things technology and the intelligent security system can realize a comprehensive safety management and security protection, so that the safety management organization can monitor all the safety control points in all directions. Based on the author's learning and practical experience, this paper first analyzed the integration of intelligent security system, then discussed the system integration framework of intelligent security of the Internet of things is discussed, then discussed the integrated framework of the intelligent security system of the Internet of things, finally put forward the specific application of intelligent security system based on Internet of things.
\end{abstract}

\section{Introduction}

Home safety is the basis for people to live in peace and contentment, and is also an important part of building a harmonious society. With the improvement of living standards, people have higher requirements for the safety and manageability of the living environment. The traditional communication of security system mostly adopts wired connection mode, which is complicated, easy to corrode and inconvenient to maintain. In recent years, the Internet of things technology has been widely used in the home security system, but most of the application systems are only preliminary, without intelligent monitoring and remote control. This paper designed a smart home security system, which integrated wireless sensor network technology, GPRS and Web technology. It can use PC machine to remotely access control or access by mobile phone. The whole system can be controlled directly by PC when the user is indoors. When you go out, you can monitor the system in real time through the network, moreover, when the indoor environment is abnormal, the system can automatically execute the short message alarm program and send the alarm short message to the user's mobile phone, so as to realize the real intelligence, we think that it has a very good prospects for development.

\section{Integration of Intelligent Security System}

Security monitoring and control system is a comprehensive technical guard system designed for the purpose of ensuring security. After several ten years of development, the development of security monitoring technology is gradually mature and widely used. The existing security system mainly includes: video surveillance system, alarm system, patrol system and intercom system. The existing security system mainly includes: video surveillance system, alarm system, patrol system and intercom system.

In some large scale security systems, video surveillance systems, burglar alarm systems and access control systems have been integrated. Related scholars give an integrated scheme of anti-theft alarm system and access control intercom system. Based on biometrics technology, they integrate effectively the anti-theft alarm and access control intercom and realize the linkage between the two subsystems. Some documents put forward a digital security solution based on HUS digital security integration platform, which realizes video surveillance, anti-theft alarm, access control and seamless integration of the third party system. In the security system platform, the synergetic 
analysis of information collected by various kinds of detectors is the key to realize intelligent handling of abnormal events. There are many kinds of detectors, including infrared detector, smoke detector, ground wave sensor and camera. Although these detectors are of different kinds, they can be classified into two categories according to their different ways of alarm for abnormal events: one is automatic supervision, the other is artificial supervision. The method of automatic supervision is that the detector automatically generates alarm signals for abnormal events, and the detection results are clear, such as infrared detectors, smoke detectors, etc. Manual supervision requires real-time monitoring of detection information, and detectors cannot automatically judge abnormal events.

The above integration scheme implements physical integration of two or more subsystems. However, these schemes only connect existing subsystems, and do not make in-depth analysis for the information gathered by subsystems. The goal of system integration is to realize centralized control and monitoring of subsystems based on a unified platform, and make corresponding linkage responses through comprehensive analysis of the information generated by subsystems. Among them, information extraction, sharing and intelligent processing are the key points of system integration. The information sharing and intelligent processing of video monitoring system can be studied by cloud computing technology, so the centralized storage and access control of video information are realized. The disadvantage is that it failed to make further in-depth analysis for the collected information by using the powerful computing function of cloud computing, and has not fully considered the integration of multiple subsystems. Studying on the integration of networking and intelligent security system, using networking technology, centralized operation and management of various security subsystem, can achieve joint interoperability between systems, so that each subsystem "connection of objects", the scope of application can not only expand the Internet of things, which can promote the development of networking technology, to improve the bottleneck in the development of the Internet of things, so it not only play a role, but also can improve further the scope of application of intelligent security system.

\section{The Integrated Framework of the Intelligent Security System of the Internet of Things}

In order to realize the integration of each subsystem effectively, this paper used hierarchical design ideas - the security system integration framework is divided into 5 layers, from bottom to top: Perceived execution layer, data aggregation layer, network connection layer, intelligent processing layer, application decision layer. The concrete frame structure is shown in Figure 1.

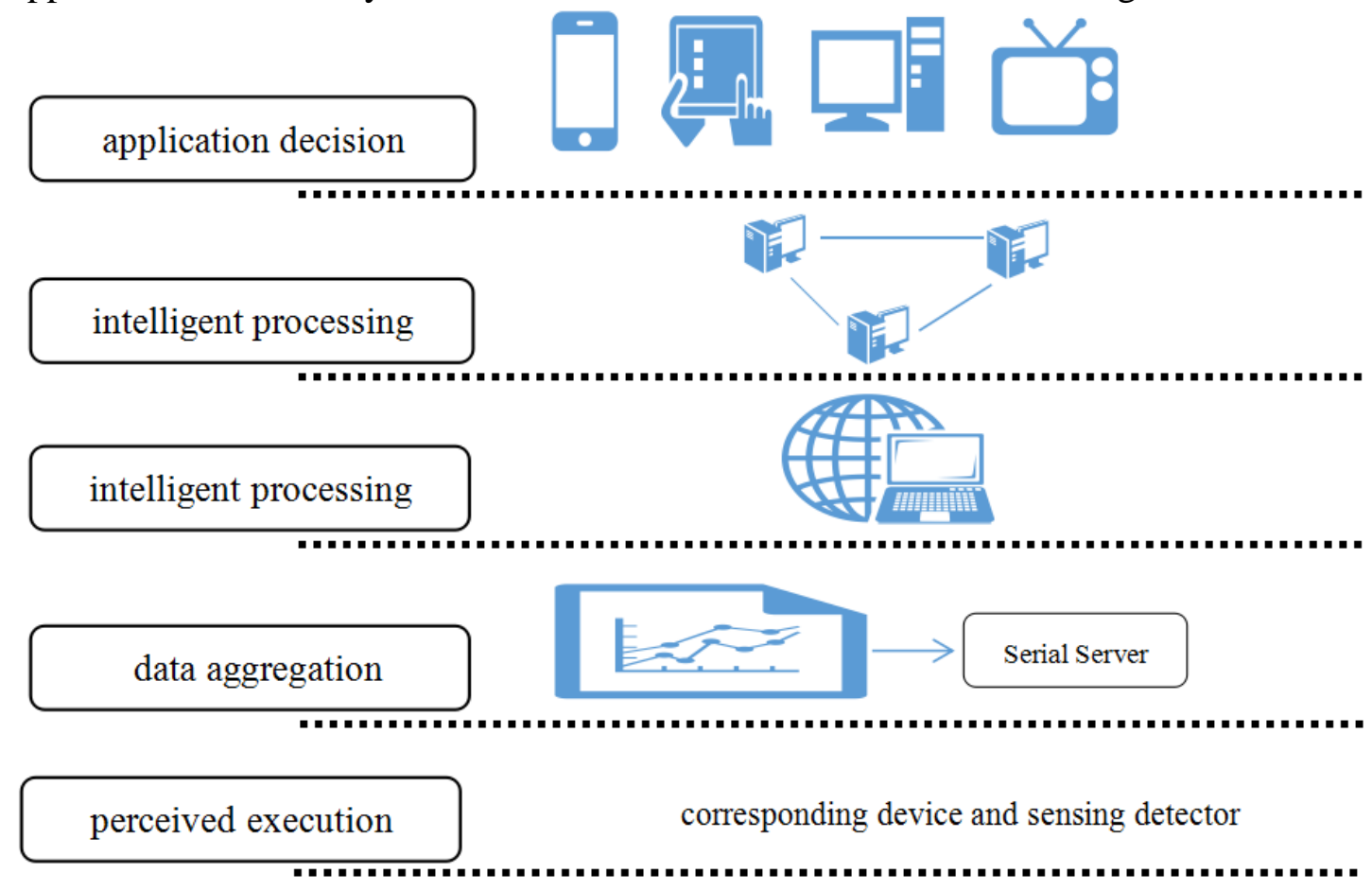

Figure 1 Integrated framework diagram of security monitoring and control system 
Perceptual execution layer: the function of perception is to collect specific information of the monitoring area through a detector. The sensing ability of the detector is the key factor affecting the performance of the security system. The equipment mainly includes: camera, smoke, infrared, vibration and other alarm detectors. Each detector has different perception and ability. In the mixed use, we need to consider the characteristics of each product so as to facilitate the linkage between the systems. The main function of execution is to respond to system control information. The commonly used execution devices include: alarm phone, short message, pan tilt, lens and LED display. Executive operation is the command of every system, rather than a single system instruction.

Data aggregation layer: compared to early data aggregation, the main difference is that the management hosts of heterogeneous subsystems are connected in accordance with different interface standards. For the upper level, the differences between the subsystems are shielded to make the upper layer transparent to the lower level. The existing control subsystem are host to provide a unified interface in accordance with the industry standard (RS232 / RS485, USB), and part of the sub system also provides IP, ZigBee and WiFi interface, so it can access through different ways to control the host access network or Internet network.

Network connection layer: the network connection layer is the medium of the detector data and control information transmission. It realizes the long-distance data transmission by utilizing the wide area connection ability of Internet network.

Intelligent processing layer: intelligent processing layer is the core of the whole system integration framework. Its main functions include data storage and collaborative analysis of multi-source data. By deploying the intelligent analysis and processing module on the server, the alarm information of a single detector can be used. Calling the corresponding function module, the information collected by other detectors in the same area is analyzed synthetically to realize the intelligent judgment of abnormal events and the comprehensive analysis ability of intelligence. It is also in sharp contrast to earlier single data processing.

Application decision layer: the main function of the application decision layer is to realize the remote monitoring and management of the system. The data is made based on the comprehensive analysis, so the execution results will be more accurate and effective. Users can monitor and manage remote monitoring terminals, such as smartphones, tablet computers, and so on, and make decisions on abnormal events in time. "More intelligent, more accurate, more convenient and more effective", this is the embodiment of the superiority of the combination of the Internet of things technology and the intelligent security system.

\section{Specific Application of Intelligent Security System Based on Internet of things}

\subsection{Intelligent Video Surveillance System based on Internet of Things}

The intelligent video surveillance system based on the Internet of things is to incorporate video camera, radio frequency equipment and radar equipment into the security and control network, to expand the scope of security detection and detect all weather conditions for different scenes. Among them, the sensor equipment has a high-definition network camera, and it is an important device to get the influence of HD monitoring. After analyzing all kinds of data through intelligent analysis system, it can make timely decisions, so as to improve the intellectualization level of video surveillance system, and improve its safety and reliability.

\subsection{Intelligent RFID access control system based on Internet of things}

With the development of information technology, the RFID entrance guard system is set up in the community and campus, after receiving the radiofrequency signal sent by the antenna, the reader can get the relevant information from the tag. RFID tags have many advantages, such as small size, easy to carry, not easy to damage, and difficult to forge. Therefore, RFID access control system can be used in different places to monitor the safety of incoming and outgoing personnel and objects. 


\subsection{Intelligent face recognition system based on Internet of things}

Combining the technology of intelligent face recognition with the technology of Internet of things, it is connected with the computer, and uses the camera on the computer to scan the image of the human face, then the scanned image is compared with the pre recorded face image in the system, and the system will automatically recognize the image. There are many technologies in the intelligent face recognition system, including facial scanning technology, feature analysis technology and neural network scanning technology. The intelligent face recognition system is mainly used for some places which are not open to public. The intelligent face recognition system consists of 4 parts: face image acquisition, face detection and location, face image normalization and face image analysis. The intelligent face recognition system can be used in community banks, key laboratories and other places.

\section{References}

[1] Zhang Weifang, Zhang Lun. Application of Internet of things technology in intelligent home control system[J]. Internet of things technology, 2015, (08): 52-53+56.

[2] Cheng Chun, Liu Yang. The application of Internet of things technology in intelligent transportation[J]. Shandong industrial technology, 2015, (07): 142-143.

[3] Yang Qiyao, Ge Quanbo. Application of internet of things technology in intelligent wireless fire fighting system[J]. Fire Science and Technology: 552-555.

[4] Gao Jianhua, Hu Zhenyu. The application of Internet of things technology in intelligent building[J]. Architecture technology, 2013, (02): 136-137.

[5] Wu Shitao. Research on the application of intelligent security system based on Internet of things technology[J]. Information security and technology, 2012, (02): 73-74+85.

[6] Wang Juan. Application of Internet of things technology in intelligent residential area[J]. Intelligent building electrical technology, 2011, (03): 94-96. 